\title{
Regadenoson use for stress myocardial perfusion imaging in advance chronic kidney disease and dialysis: Safe, effective, and efficient
}

\author{
Ankur Gupta, MD, PhD, a and Navkaranbir S. Bajaj, MD, $\mathrm{MPH}^{\mathrm{a}}$ \\ a Division of Cardiovascular Medicine and Department of Radiology, Brigham and Women's \\ Hospital, Harvard Medical School, Boston, MA
}

Received Aug 4, 2017; accepted Aug 4, 2017

doi: 10.1007/s12350-017-1038-1

\section{See related article, pp. 137-149}

The pharmacological vasodilator stress agents used for clinical stress myocardial perfusion imaging include adenosine, dipyridamole, and regadenoson. Adenosine is a non-selective stress agent that leads to coronary vasodilation by activating $\mathrm{A} 2 \mathrm{~A}$ receptors on smooth muscle cells. However, it also activates A1 receptors in sinus and atrioventricular nodes, and atrial and ventricular myocytes with associated negative chronotropic, dromotropic, and inotropic effects in addition to inducing chest pain in over one-third of the patients. ${ }^{1-3}$ Dipyridamole, by inhibiting adenosine degradation, leads to increased adenosine levels at its receptor sites and thus, has a safety and tolerability profile comparable with adenosine. ${ }^{4}$ In contrast, regadenoson is a selective A2A receptor antagonist. This selective mechanism of action has led to a significantly better tolerability profile of regadenoson compared with adenosine with similar safety profile. $^{2}$

Regadenoson also provides diagnostic and prognostic information comparable to adenosine. ${ }^{2,5,6}$ Further, in contrast to adenosine, regadenoson is not rapidly metabolized by plasma adenosine deaminase or cell membrane nucleoside transporter. This allows intravenous bolus administration of regadenoson rather than

Funding: Drs. Gupta and Bajaj are supported by NIH Grant No. 5T32HL094301.

Reprint requests: Ankur Gupta, MD, PhD, Division of Cardiovascular Medicine and Department of Radiology, Brigham and Women's Hospital, Harvard Medical School, ASB-L1 037C, 75 Francis Street, Boston, MA, 02115; agupta@bwh.harvard.edu

J Nucl Cardiol 2018;25:150-2.

$1071-3581 / \$ 34.00$

Copyright (C) 2017 American Society of Nuclear Cardiology. continuous intravenous drip required by adenosine. ${ }^{1}$ These pharmacokinetic properties of regadenoson, therefore, allow for efficient work flow in the nuclear stress laboratories. Hence, with its comparable effectiveness and safety and improved tolerability and work flow efficiency, it is no surprise that soon after the U.S. Food and Drug Administration's (FDA) approval of regadenoson for use as a stress agent in myocardial perfusion imaging in 2008, there was rapid adoption of it as the preferred stress agent in nuclear laboratories across the U.S., capturing $84 \%$ of the market by $2013 .{ }^{7}$

Unlike adenosine or dipyridamole, approximately $58 \%$ of regadenoson elimination occurs through kidneys. ${ }^{8}$ This led to the concerns of safety and tolerability of regadenoson in patients with impaired renal function. Prior to the 2008 FDA approval of regadenoson use as a stress agent, Gordi et al. ${ }^{9}$ studied pharmacokinetics across a spectrum of patients with normal renal function or chronic kidney disease (CKD) without end-stage renal disease (ESRD) on dialysis. They found that although patients with CKD had higher elimination halflife of regadenoson compared with controls with normal renal function, the maximum observed plasma concentration of regadenoson was similar to that of controls. ${ }^{9}$ This led to the FDA outlining the regadenoson use in CKD patients without dose adjustment in its 2008 approval. However, at that time, there were no clinical studies on safety or tolerability of regadenoson in CKD patients.

Further, there were no data regarding pharmacokinetics, safety, or tolerability of regadenoson in patients with ESRD on dialysis. The 2008 FDA approval reflected this with regadenoson label indicating that its pharmacokinetics in ESRD patients on dialysis has not been assessed. ${ }^{10}$ This was followed by a nearly nineyear hiatus in the on-label use of regadenoson in ESRD patients. Ultimately, in a label update in January 2017, the FDA has outlined the use of regadenoson in ESRD 
patients and those on dialysis. ${ }^{11}$ The label now indicates that no dose adjustment is needed in patients with renal impairment including those with ESRD and/or dependent on dialysis. ${ }^{11}$

In this issue of the Journal, Vij et al. ${ }^{12}$ provide a very timely review of the evidence leading up to the label update including studies of pharmacokinetics, safety, tolerability, and diagnostic and prognostic utility of regadenoson in patients with CKD and ESRD on dialysis. Although no in vivo studies address pharmacokinetics of regadenoson in ESRD patients, an in vitro study $^{13}$ showed that hemodialysis results in modest clearance of regadenoson relative to total body clearance and is unlikely to produce a clinical significant outcome. Two large observational studies in patients with ESRD established that the safety, tolerability, and hemodynamic profiles of regadenoson in these patients is comparable to those with normal or mild to moderately impaired renal function. ${ }^{14,15}$ Further in addition to an observational study, ${ }^{16}$ randomized double blind placebo controlled trial evidence conclusively established

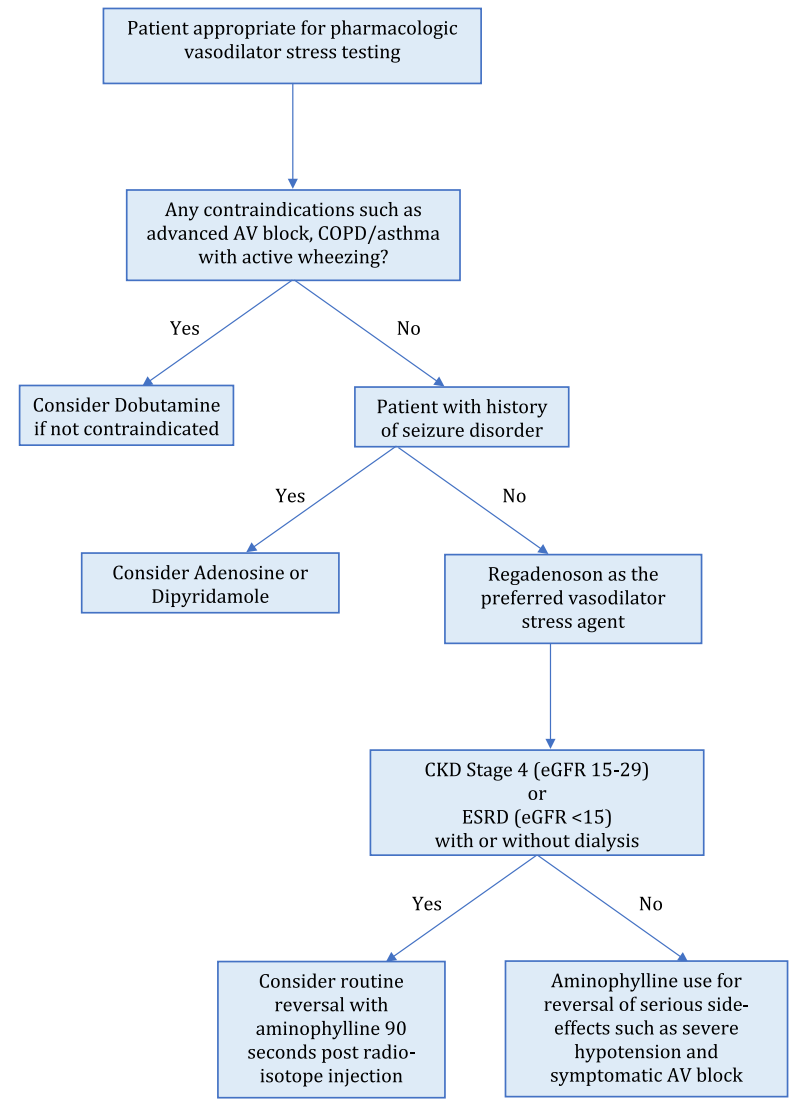

Figure 1. Choosing stress agent for pharmacological stress myocardial perfusion imaging. AV, atrioventricular; CKD, chronic kidney disease; COPD, chronic obstructive pulmonary disease; eGFR, estimated glomerular filtration rate; ESRD, endstage renal disease. eGFR is expressed in $\mathrm{mL} / \mathrm{min} / 1.73 \mathrm{~m}^{2}$. the safety of regadenoson in CKD 3-4 (estimated glomerular filtration rate, $\mathrm{eGFR}=15-60 \mathrm{~mL} / \mathrm{min} / 1.73 \mathrm{~m}^{2}$ ) patients. $^{17}$

Aminophylline reversal of the adverse effects of vasodilatory stress agents could play a particularly important role in the use of regadenoson in patients with CKD and ESRD. The ASSUAGE-CKD trial ${ }^{18}$ investigating the routine use of aminophylline in patients with CKD $4\left(\mathrm{eGFR}=15-29 \mathrm{~mL} / \mathrm{min} / 1.73 \mathrm{~m}^{2}\right)$ and ESRD found two-third reduction in incidence of diarrhea (from $17 \%$ to $6 \%$ ) and headache (from $36 \%$ to $11 \%$ ), and onehalf reduction in any regadenoson-related adverse effects (from $64 \%$ to $31 \%$ ), irrespective of age, sex, ethnicity, CKD stage 4 or 5, and without any impairment in myocardial perfusion imaging sensitivity for the detection and extent of perfusion deficits. ${ }^{19}$

In clinical use of pharmacologic vasodilatory stress agents, regadenoson will remain the workhorse and routine reversal with aminophylline could further improve its tolerability in patients with advance CKD and ESRD, Figure 1. The 2017 FDA label update will further streamline the use of regadenoson in a majority of patients undergoing stress myocardial perfusion imaging, improving work-flows and efficiency of nuclear cardiology laboratories, and ultimately benefitting our patients by provision of evidence-based care.

\section{Disclosures}

Drs. Gupta and Bajaj have no relevant disclosures.

\section{References}

1. Gupta A, Zoghbi GJ, Hage FG. Pharmacologic stress testing. Principles and Applications: Nuclear Cardiac Imaging. Oxford: Oxford University Press; 2015. p. 196.

2. Iskandrian $\mathrm{AE}$, Bateman $\mathrm{TM}$, Belardinelli L, et al. Adenosine versus regadenoson comparative evaluation in myocardial perfusion imaging: results of the ADVANCE phase 3 multicenter international trial. J Nucl Cardiol. 2007;14:645-58.

3. Cerqueira MD, Verani MS, Schwaiger M, Heo J, Iskandrian AS. Safety profile of adenosine stress perfusion imaging: results from the Adenoscan Multicenter Trial Registry. J Am Coll Cardiol. 1994;23:384-9.

4. Ranhosky A, Kempthorne-Rawson J. The safety of intravenous dipyridamole thallium myocardial perfusion imaging. Intravenous Dipyridamole Thallium Imaging Study Group. Circulation. 1990;81:1205-9.

5. Cerqueira MD, Nguyen $P$, Staehr P, Underwood SR, Iskandrian AE. Investigators A-MT. Effects of age, gender, obesity, and diabetes on the efficacy and safety of the selective A2A agonist regadenoson versus adenosine in myocardial perfusion imaging integrated ADVANCE-MPI trial results. JACC Cardiovasc Imaging. 2008;1:307-16.

6. Mahmarian JJ, Peterson LE, Xu J, et al. Regadenoson provides perfusion results comparable to adenosine in heterogeneous 
patient populations: a quantitative analysis from the ADVANCE MPI trials. J Nucl Cardiol. 2015;22:248-61.

7. American Society of Nuclear Cardiology / MedAxiom Nuclear Survey 2013. J Nucl Cardiol 2014;21 Suppl 1:5-88.

8. Gordi T, Frohna P, Sun HL, Wolff A, Belardinelli L, Lieu H. A population pharmacokinetic/pharmacodynamic analysis of regadenoson, an adenosine A2A-receptor agonist, in healthy male volunteers. Clin Pharmacokinet. 2006;45:1201-12.

9. Gordi T, Blackburn B, Lieu H. Regadenoson pharmacokinetics and tolerability in subjects with impaired renal function. J Clin Pharmacol. 2007;47:825-33.

10. Center for drug evaluation and research. Application number 22161. Labeling. https://www.accessdata.fda.gov/drugsatfda_docs/ nda/2008/022161s000_LEXISCAN_PRNTLBL.pdf. Accessed 2 Aug 2017

11. Astellas Pharma U.S. Lexiscan (Regadenoson) Highlights of Prescribing Information. [updated January 2017]. http://www.astellas. us/docs/lexiscan.pdf. Accessed 2 Aug 2017.

12. Vij A, Golzar Y, Doukky R. Regadenoson use in chronic kidney disease and end-stage renal disease: a focused review. J Nucl Cardiol. 2017; doi:10.1007/s12350-017-0960-6.

13. Gharibian KN, Murthy VL, Mueller BA. Influence of hemodialysis on regadenoson clearance in an in vitro hemodialysis model. J Nucl Cardiol. 2016;. doi:10.1007/s12350-016-0667-

14. Aljaroudi W, Hermann D, Hage F, Heo J, Iskandrian AE. Safety of regadenoson in patients with end-stage renal disease. Am J Cardiol. 2010;105:133-5.
15. Doukky R, Rangel MO, Wassouf M, Dick R, Alqaid A, Morales Demori R. The safety and tolerability of regadenoson in patients with end-stage renal disease: the first prospective evaluation. $\mathrm{J}$ Nucl Cardiol. 2013;20:205-13.

16. Palani G, Husain Z, Salinas RC, Karthikeyan V, Karthikeyan AS, Ananthasubramaniam K. Safety of regadenoson as a pharmacologic stress agent for myocardial perfusion imaging in chronic kidney disease patients not on hemodialysis. J Nucl Cardiol. 2011;18:605-11.

17. Ananthasubramaniam K, Weiss R, McNutt B, Klauke B, Feaheny $\mathrm{K}$, Bukofzer S. A randomized, double-blind, placebo-controlled study of the safety and tolerance of regadenoson in subjects with stage 3 or 4 chronic kidney disease. J Nucl Cardiol. 2012;19:31929.

18. Doukky R, Rangel MO, Dick R, Wassouf M, Alqaid A, Margeta B. Attenuation of the side effect profile of regadenoson: a randomized double-blind placebo-controlled study with aminophylline in patients undergoing myocardial perfusion imaging and have severe chronic kidney disease-the ASSUAGE-CKD trial. Int J Cardiovasc Imaging. 2013;29:1029-37.

19. Fughhi I, Campagnoli T, Ali A, Doukky R. Impact of a regimented aminophylline administration protocol on the burden of regadenoson-induced ischemia detected by SPECT myocardial perfusion imaging. J Nucl Cardiol. 2016; d doi:10.1007/s12350-016-0506-. 